\title{
Ion and Electron Cyclotron Wall Conditioning in Stellarator and Tokamak Magnetic Field Configuration on WEGA
}

\author{
T. Wauters*, H. Laqua ${ }^{\dagger}$, M. Otte ${ }^{\dagger}$, M. Preynas ${ }^{\dagger}$, T. Stange ${ }^{\dagger}$, P. Urlings ${ }^{\dagger, * *}$, Y. \\ Altenburg $^{\dagger}$, D. Aßmus ${ }^{\dagger}$, D. Birus ${ }^{\dagger}$ and F. Louche ${ }^{\dagger}$ \\ ${ }^{*}$ Association Euratom-Belgian State, LPP-ERM/KMS, 1000 Brussels, Belgium \\ ${ }^{\dagger}$ Max Planck Institute for Plasma Physics, EURATOM Association, 17491 Greifswald, Germany \\ ${ }^{* *}$ University of Technology, Department of Applied Physics, Eindhoven
}

\begin{abstract}
Discharge wall conditioning is an effective tool to improve plasma performance in tokamaks and stellarators. RF Discharge Conditioning (RFDC) techniques are envisaged for use during operational campaigns on superconducting devices like the ITER tokamak and W7-X stellarator, as alternative to DC Glow Discharge Conditioning, which is inefficient in presence of magnetic fields. This contribution investigates RFDC in both the ion and electron cyclotron range of frequencies (ICRF and ECRF) on the WEGA device (Max-Planck-Institute for Plasma Physics, Greifswald, Germany) as preparation for W7-X operation.

ECRF discharges produced by localised absorption of RF power at EC resonance layers suffer from poor radial discharge homogeneity in the tokamak vacuum magnetic field configuration, severely limiting the plasma wetted wall areas and consequently the conditioning efficiency. The non-localised production of ICRF discharges by collisional RF power absorption features much improved discharge homogeneity making Ion Cyclotron Wall Conditioning (ICWC) the favoured RFDC technique for superconducting tokamaks. RFDC with the stellarator vacuum magnetic field needs to aim at sufficient plasma densities at and outside the LCFS, maximising the convective plasma flux along the open field lines to the wall. Whereas for ICRF discharges this condition is easily fulfilled, on WEGA for He-ECRF discharges this could be achieved as well by off axis heating close to the LCFS.

In stellarator magnetic field configuration it is found that He-ICWC for wall desaturation is at least one order of magnitude more efficient than He-ECWC. Novel ECWC methods are proposed that can decrease this efficiency gap with ICWC gap to a factor 2-3. The efficiency difference is less pronounced in case of $\mathrm{H}_{2}$-ICWC and ECWC for isotopic exchange.
\end{abstract}

Keywords: RF discharge, ICRF, ECRF, Wall conditioning, Tokamak, Stellarator

PACS: 52.50.-b, 52.50.Qt, 52.50.Sw, 52.55.Fa, 52.55.Hc, 52.77. Bn, 52.80.Pi

\section{INTRODUCTION}

Discharge wall conditioning is an effective tool to improve plasma performance in tokamaks and stellarators by (i) reducing the generation of plasma impurities liberated from the wall and (ii) controlling the recycling of hydrogenic fluxes [1]. RF Discharge Conditioning (RFDC) techniques are envisaged for use during operational campaigns on superconducting devices like the ITER tokamak and W7-X stellarator, as alternative to DC Glow Discharge Conditioning, which is inefficient in presence of magnetic fields (see e.g. [2]). This contribution investigates RFDC in both the ion and electron cyclotron range of frequencies (ICRF and ECRF) on the WEGA device (Max-Planck-Institute for Plasma Physics, Greifswald, Germany) as preparation for W7-X operation. The dependencies of both RFDC techniques on discharge parameters such as magnetic field configuration (toroidal field and rotational transform), pressure and power are discussed, and the efficiency for wall desaturation $(\mathrm{H}$ removal) and isotope exchange are compared. Detailed analysis of ECRF and ICRF discharge initiation can be found respectively in [3,4].

\section{EXPERIMENTAL SETUP}

WEGA in Greifswald is a flexible Hybrid-experiment (both tokamak and stellarator operation is possible) used for educational purposes, diagnostic development, research on wave heating and transport in plasmas and as testbed for prototype control system of W7-X [5]. The metallic device with major radius $R=72 \mathrm{~cm}$ and minor radius $r=19 \mathrm{~cm}$ was for this RFDC study operated at magnetic fields of up to $0.5 \mathrm{~T}$ with rotational transform ranging from $l=0$ to 0.37 . The employed plasma heating systems consist of a $10 \mathrm{~kW} 28 \mathrm{GHz}$ gyrotron for $\mathrm{X} 2$ mode electron heating 


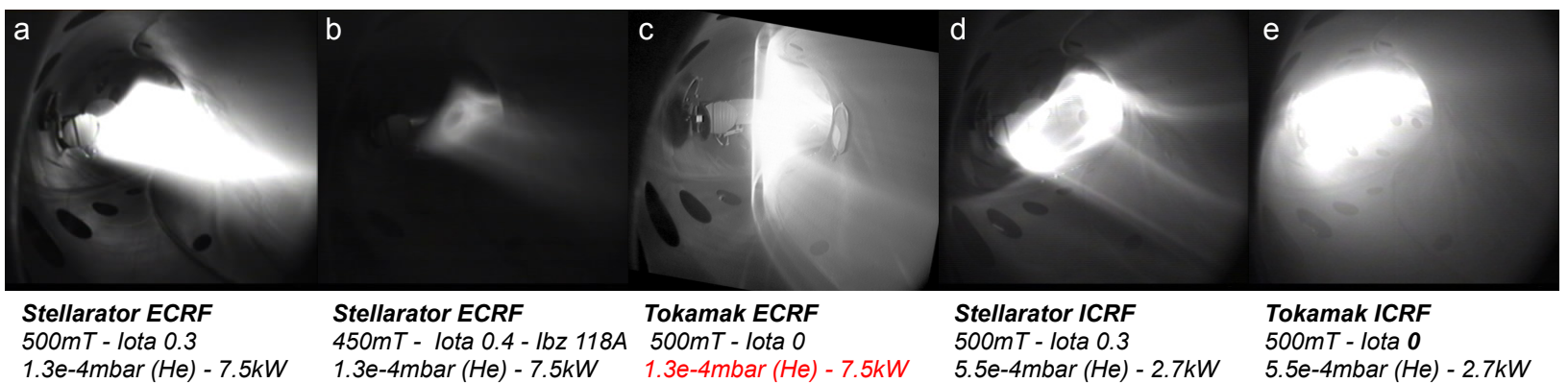

FIGURE 1. CCD camera images (tangential view) for ECRF and ICRF discharges in stellarator and tokamak magnetic field configuration on the WEGA device.

with second harmonic at $0.5 \mathrm{~T}$ with its beam oriented nearly parallel to the poloidal plane, a $6-20 \mathrm{~kW} 2.45 \mathrm{GHz}$ magnetron resonant at low magnetic field, and a (for this study devised) single loop ICRF antenna without antenna box tuneable at 6 and $9 \mathrm{MHz}$, for which the latter has the fundamental $\mathrm{H}^{+}$resonance at $R=0.6 \mathrm{~m}$ for $0.5 \mathrm{~T}$ (HFS). The diagnostics used for this contribution are mass spectrometry and ionisation gauges for (partial) pressures, gas flow meters for monitoring gas injection rates, RF power related signals, CCD camera images for visual plasma inspection, interferometry $(80 \mathrm{GHz})$ for line integrated electron density and a Langmuir probe for density and temperature.

\section{DISCHARGE CHARACTERISATION AND OPTIMISATION}

Typical characteristics of ECRF and ICRF discharges on stellarators and tokamaks as observed by CCD images for the visible spectrum are depicted in Figure 1. ECRF discharges produced by localised absorption of RF power at EC resonance condition suffer from poor radial discharge homogeneity in the tokamak vacuum magnetic field configuration (Fig. 1c) well-marked by the bright vertical radiation zone around the resonance layer ${ }^{1}$. The hence severely limited plasma wetted wall area, excluding HFS and LFS, results in an equally in-homogeneously conditioned wall. Although the poloidal homogeneity can be improved by applying a small radial magnetic field component, this action introduces electron losses from the resonant area while here the density must remain well above the cutoff density for local single pass ECRF absorption $\left(n_{e, c}=\ldots \mathrm{m}^{-3}\right)$ to avoid surface melting where the ECRF bundle strikes the wall. The non-localized production of ICRF discharges by collisional RF power absorption via electrons [6] features much improved discharge homogeneity (Fig. 1e) making Ion Cyclotron Wall Conditioning (ICWC) the favored RFDC technique for superconducting tokamaks [7].

RFDC with the stellarator vacuum magnetic field needs to aim at sufficient plasma densities at and outside the LCFS, maximizing the convective plasma flux along the open field lines to the wall. For ICRF discharges this condition is maximally fulfilled, illustrated by Fig. $1 \mathrm{~d}\left(n_{e, l}=1.5 \times 10^{16} \mathrm{~m}^{-2}\right)$. The plasma extends into the confined area while the brightest radiation zone locates at the LCFS exposing the structure of magnetic islands in the edge. On-axis heating in ECRF discharges on WEGA however produces a center-peaked density profile $\left(n_{e, l}=3.3 \times 10^{17} \mathrm{~m}^{-2}\right)$ which is poorly connected to the wall and is as such considered not suitable for conditioning (Fig. 1a). An improved ECDC scenario increasing the edge density and as such the convective plasma flux to the wall was developed employing offaxis heating close to the LCFS (Fig. 1b), obtained by operating at slightly reduced magnetic field ( $0.45 \mathrm{~T}$ ), equivalent to tilting beam mirrors in the poloidal plane. A hollow density profile was observed $\left(n_{e, \max }=6 \times 10^{17} \mathrm{~m}^{-3}, T_{e, \min }=\right.$ $2.4 \mathrm{eV} @ r=2.5 \mathrm{~cm})$ with much reduced electron density $\left(n_{e, l}=1.5 \times 10^{16} \mathrm{~m}^{-2}\right)$ due to the larger power deposition volume. Again, for ECDC applications based on this regime e.g. on W7-X, care must be taken to remain well above the cut-off density for single pass absorption by compensating the ERCF power level. The final optimised gyrotron ECRF discharge scenario for wall conditioning used slightly off-axis heating together with an iota sweep $(l=0.37-0.25)$ to increase the conditioning homogeneity by moving the plasma strike points. On superconducting W7-X such sweep may be reproduced by employing the normal conducting trim coils or by slowly changing the field configuration from

\footnotetext{
${ }^{1}$ For a similar setup (X2-mode) with more tangential beam angle, discharges concentrated around the ECRF beam were reported in [7] (TEXTOR).
} 

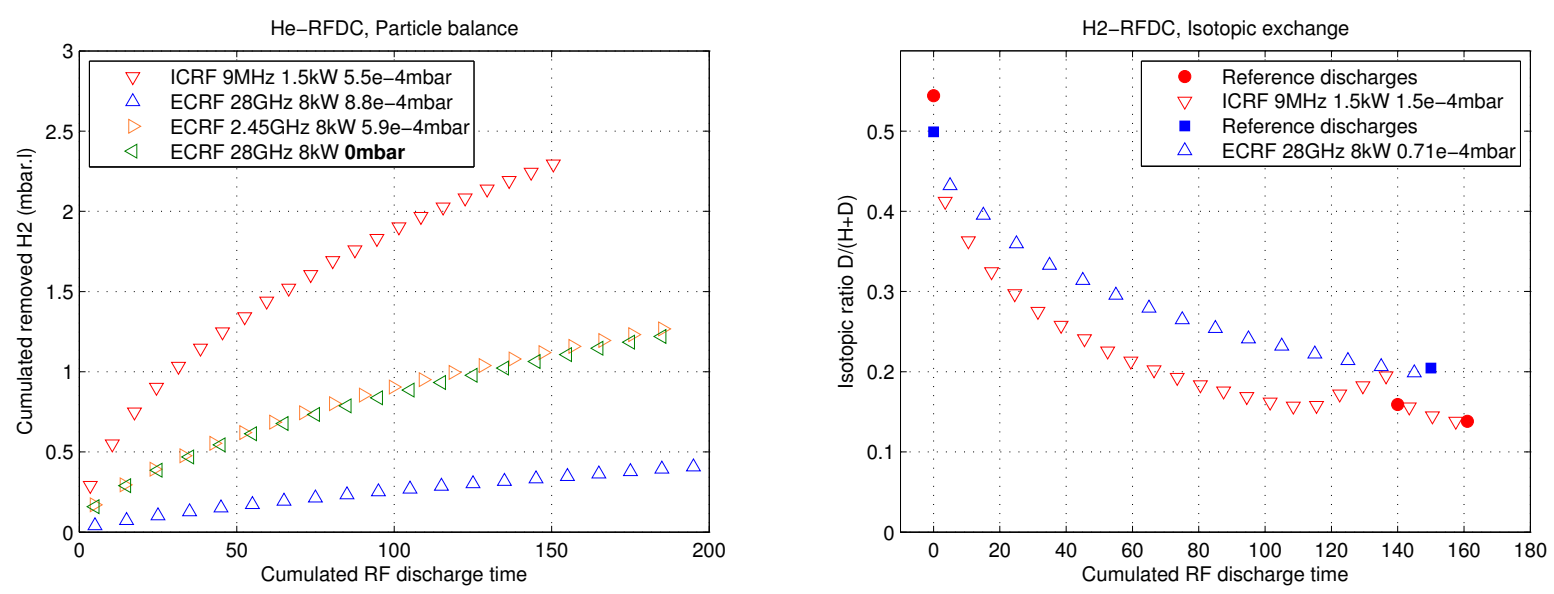

FIGURE 2. Comparison of wall conditioning efficiency for 4 different RFDC techniques on WEGA device. Left: Wall desaturation by He-ICWC and He-ECWC, non-resonant He-ECRF discharges and ECRF discharges without gas feed. Right: Isotopic exchange by $\mathrm{H}_{2}$-ICWC and $\mathrm{H}_{2}$-ECWC.

pulse to pulse in multi-pulse ECWC operation.

\section{WALL CONDITIONING EFFICIENCY}

The wall desaturation and isotopic exchange efficiency of Ion and Electron Cyclotron Wall Conditioning are compared for stellarator magnetic field configuration. The procedure for wall desaturation consisted of (a) reference gyrotron discharges without gas injection (only wall fuelling) to sample the wall hydrogen content via the neutral $\mathrm{H}_{2}$-pressure during these discharges, (b) series of $25 \mathrm{~s} \mathrm{H}_{2}$-magnetron discharges at low magnetic field to load the wall with approx. $5.50 \mathrm{mbar} .1$ of molecules until a stable $\mathrm{H}_{2}$-pressure level during the discharge was obtained, and (c) series of $\sim 20$ optimised conditioning discharges of the to-be-compared RFDC techniques, namely: (i) He-ICWC, (ii) HeECWC, (iii) He-magnetron discharges, and (iv) gyrotron discharges without gas injection, all at nominal magnetic field of $0.5 \mathrm{~T}$ and fixed iota $\imath=0.3$, except for He-ECWC employing an iota scan. The magnetron discharges were initiated by a $3 \mathrm{~s}$ gyrotron pulse at $0.5 \mathrm{~T}$ after which the non-resonant magnetron frequency sustained the discharge over $9.5 \mathrm{~s}$. Non-resonant magnetron discharges with ICRF assisted start-up are found also feasible.

The reference $\mathrm{H}_{2}$-pressure before the first $\mathrm{H}_{2}$-inlet was $p_{\mathrm{H}_{2} \text {,ref }}=2.8 \times 10^{-7} \mathrm{mbar}$. The wall loading procedure allowed to obtain a reproducible wall state before each series of conditioning discharges evidenced by the small deviation on the reference pressure after loading: $p_{\mathrm{H}_{2}, \text { ref }}=1.7 \pm 0.1 \times 10^{-5}$ mbar. It is found that only He-ICWC completely recovered the initial wall state: $p_{\mathrm{H}_{2}}=2.7 \times 10^{-7}$ mbar, while the He-ECWC procedure of similar duration attained a $\mathrm{H}_{2}$-reference pressure level of $p_{\mathrm{H}_{2}}=4.4 \times 10^{-6} \mathrm{mbar}$, still more than an order of magnitude above the initial value. This result is reflected in Figure 2a showing the recovered amount of $\mathrm{H}_{2}$-molecules as a function of cumulated RF discharge time. After $t_{\mathrm{RF}}=150 \mathrm{~s}$ the four techniques (i, ii, iii, and iv) have recovered respectively 42, 6, 21 and $20 \%$ of the wall loaded $\mathrm{H}_{2}$. For the tested time range the curves show no sign of saturation. The plot as function of cumulated RF discharge time for the discharges of different durations (resp. 7.5, 10, 9.5 \& 10s) is representative as the removal scales close to linearly with discharge length. It should also be noted that the lower coupled power level for ICWC of $1.5 \mathrm{~kW}$, due to the RF generator power limit together with $\sim 50 \%$ coupling efficiency, compared to $8 \mathrm{~kW}$ for ECWC in this comparison, puts the EC-RFDC technique in advantage as it is known that the removal efficiency for RFDC increases approximately linear with increasing coupled power [8]. Weighted by power level, ICWC is then estimated $\sim 50$ times more efficient than ECWC. The hydrogen removal efficiency of He-ICWC is found to be weakly dependent on the discharge pressure in the range of $1-10 \times 10^{-4} \mathrm{mbar}$, as the pressure dependency is masked by pulse to pulse ongoing desaturation of wall. For He-ICWC with off-axis heating and iota sweep the efficiency was higher in case of higher gas throughput.

The higher efficiency of gyrotron discharges without gas injection (iv) compared to gyrotron discharges with Heinjection (ii) is accredited to the stellarator confining field and requires further study. It is thought to originate from the improved connection along open field lines between the confined center plasma and the wall in case of the discharges 
without gas injection, as lower neutral pressure allows for lower collisionallity. It is also known that wall desorbed hydrogen contributes significantly to the wall flux in RFDC plasmas [9] (high recycling), and that the major hydrogen flux consists of neutral hydrogen atoms produced upon dissociation [10], which are not confined by the magnetic field. The higher efficiency of He-magnetron discharges (iii) compared to He-gyrotron discharges (ii) is due to the improved discharge homogeneity and wall coverage resulting from non-resonant electron collisional RF power absorption in case of magnetron frequency $2.45 \mathrm{GHz}$ at $0.5 \mathrm{~T}$.

The procedure for isotopic exchange consisted of (a) reference gyrotron discharges without gas injection (only wall fuelling) to sample the isotopic ratio of the wall via the partial $\mathrm{H}_{2}, \mathrm{HD}$ and $\mathrm{D}_{2}$-pressure during these discharges, (b) series of $25 \mathrm{~s} \mathrm{D}_{2}$-magnetron discharges at low magnetic field to load the wall up until a isotopic ratio of approx. $[\mathrm{D}] /[\mathrm{H}+\mathrm{D}]=0.5$, and (c) series of $\sim 20$ optimised (i) $\mathrm{H}_{2}$-ICWC and (ii) $\mathrm{H}_{2}$-ECWC discharges at nominal magnetic field of $0.5 \mathrm{~T}$. Figure $2 \mathrm{~b}$ shows that the difference between ICWC and ECWC is not as pronounced as for wall desaturation. In $150 \mathrm{~s}$ of cumulated RF discharge time the isotopic ratio could be changed by $73 \%$ in case of $\mathrm{H}_{2}$ ICWC and 59\% in case of $\mathrm{H}_{2}$-ECWC. Here as well the power difference between ICWC $(\ldots \mathrm{kW})$ and ECWC $(\ldots \mathrm{kW})$ puts the latter in advantage. The slightly increasing isotopic ratio at $t_{\mathrm{RF}}=130 \mathrm{~s}$ in case of ICWC is due to the emptying of the $\mathrm{H}_{2}$ gas injection reservoir, which could be refilled after the reference pulse at $t_{\mathrm{RF}}=150 \mathrm{~s}$.

\section{CONCLUSION}

RFDC in both the ion and electron cyclotron range of frequencies (ICRF and ECRF) were investigated on the WEGA device (Max-Planck-Institute for Plasma Physics, Greifswald, Germany) as preparation for W7-X operation. ECRF discharges produced by localised absorption of the RF power at EC resonance layers suffer from poor radial discharge homogeneity in the tokamak vacuum magnetic field configuration, severely limiting the plasma wetted wall areas and consequently the conditioning efficiency. The non-localised production of ICRF discharges by collisional RF power absorption features much improved discharge homogeneity making Ion Cyclotron Wall Conditioning (ICWC) the favoured RFDC technique for superconducting tokamaks. RFDC with the stellarator vacuum magnetic field needs to aim at sufficient plasma densities at and outside the LCFS, maximising the convective plasma flux along the open field lines to the wall. Whereas for ICRF discharges this condition is easily fulfilled, on WEGA for He-ECRF discharges this could be achieved as well by off axis heating. An iota sweep moving strike points increases the wall coverage.

For the stellarator magnetic field configuration it is found that He-ICWC for wall desaturation is at least one order of magnitude more efficient than standard He-ECWC. Novel ECWC methods are proposed that can decrease the efficiency gap with ICWC gap to a factor 2, namely ECWC discharges at reduced (non-resonant) frequency allowing for more homogeneous collisional absorption of RF power aided by resonant ECRF assisted startup, or ECRF discharges at nominal frequency relying on wall fuelling only. Also for isotopic exchange $\mathrm{H}_{2}$-ICWC is found to be more efficient although here the difference to ECWC is less pronounced.

\section{ACKNOWLEDGMENTS}

This work was supported by EURATOM and carried out within the framework of the EFDA Fellowships Programme.

The views and opinions expressed herein do not necessarily reflect those of the European Commission. The authors would like to thank the WEGA team for their excellent assistance and support of this work.

\section{REFERENCES}

1. J. Winter, Plasma Phys. Control. Fusion 38, 1503-1542 (1996).

2. D. Douai et al. Proc. 20th RFPPC R4, Sorrento, 25-28 June 2013, this conference (2013).

3. M. Preynas et al. Proc. 20th RFPPC P..., Sorrento, 25-28 June 2013, this conference (2013).

4. M. Tripsky et al. Proc. 20th RFPPC P2.33, Sorrento, 25-28 June 2013, this conference (2013).

5. M. Otte et al. Proc. 17th International Stellarator and Heliotron Workshop, Princeton, 12-16 October 2009 (2009).

6. A. Lyssoivan et al. Plasma Phys. Control. Fusion 54, 074014 (2012)

7. E. de la Cal, E. Gauthier, Plasma Phys. Control. Fusion 47, 197-218 (2005).

8. R. Brakel et al. J. Nucl. Mat. 290-293, 1160-1164 (2001)

9. T. Wauters et al. J. Nucl. Mat. 415, S1033-S1036 (2011)

10. T. Wauters et al. Plasma Phys. Control. Fusion 53, 125003 (2011) 